\title{
Medical Care Burden of Children with Autism Spectrum Disorders
}

\author{
Jin-Ding Lin
}

Received: 19 May 2014 / Accepted: 23 May 2014 / Published online: 3 June 2014

(C) Springer Science+Business Media New York 2014

\begin{abstract}
This paper aims to examine health care utilization and expenditure in the provision of medical care to understand the medical care burden of children with autism spectrum disorders based on recent literature reviews. This article reviews the recent literature in Medline, PubMed, and Google by using key terms that are relevant to autism spectrum disorder (ASD) and health care (medical care) utilization, medical care costs, and expenditures. I also hand-searched the reference lists of all of the included articles and recent narrative and systematic reviews related to medical care utilization and the costs of ASD to identify potentially relevant articles. The literature on medical care utilization and expenditures related to ASD highlights the fact that the disorder imposes high medical care burdens on families and on society. It is necessary to initiate appropriate, comprehensive, and accessible medical care services for individuals with ASD, particularly for those with comorbid conditions. Future studies should examine the impact of such improvements in the management of children with ASD on medical care utilization and costs.
\end{abstract}

Keywords Autism spectrum disorders $\cdot$ Medical need · Medical utilization $\cdot$ Medical cost

J.-D. Lin $(\bowtie)$

School of Public Health, National Defense Medical Center, No. 161, Minquan E. Rd., Section 6, Nei-Hu, Taipei 114, Taiwan

e-mail: a530706@ndmetsgh.edu.tw

J.-D. Lin

Chung-Hua Foundation for Persons with Intellectual Disabilities, New Taipei City, Taiwan

J.-D. Lin

Center for Environment and Population Health, Griffith University,

Brisbane, Australia

\section{Introduction}

Autism spectrum disorder (ASD), identified by Leo Kanner in the early 1940s (Kanner 1943), is a set of heterogeneous neurodevelopmental conditions (Lai et al. 2014) that is characterized by unusual social, communication, and behavioral development. Currently, there are no genetic or biologic markers for ASD to enable the consistent and early identification of affected children (Rice et al. 2004). Generally, ASD affects more male than female individuals (Kogan et al. 2009; Lai et al. 2014). Epidemiological data estimate the global prevalence of ASD to be one person in 160, accounting for more than 7.6 million disability-adjusted life years and $0.3 \%$ of the global burden of disease (WHO 2013). The CDC's estimate comes from the Autism and Developmental Disabilities Monitoring (ADDM) Network, which monitors the number of 8-year-old children with ASD living in diverse communities throughout the USA and reported that approximately 1/150 to 1/88 children had an ASD (CDC 2007a, b, 2009, 2012). Maenner and Durkin (2010) estimated that the prevalence of the use of the autism special education category in Wisconsin appears to be leveling off in school districts, with the highest prevalence rate at 12 cases per 1,000 students. On average, approximately $1 \%$ or one child in every 110 was classified as having an $\mathrm{ASD}$, and these results indicate an increased prevalence of identified ASD among US children and underscore the need to regard ASD as an urgent public health concern (CDC 2009). The heightened prevalence of ASD is most likely attributable to increased awareness and changes in the diagnostic criteria rather than to new environmental influences (Muhle et al. 2004).

The time cost of caring for a child with severe disabilities is significantly greater than caring for a nondisabled child and does not decrease with increasing age (Curran et al. 2001). ASDs have lifetime consequences, with a range of impacts on 
the health, economic well-being, social integration, and quality of life of individuals with the disorder, as well as on their families and potentially society at large. The aggregate national costs of supporting children with ASD were estimated to be $£ 2.7$ billion each year in the UK (Foundation for People with Learning Disabilities 2007). Childhood ASD is associated with a substantial loss of annual household income (Montes and Halterman 2008). Among the health care concerns for children with ASD is a major need to strengthen the awareness and treatment of its associated medical conditions with standardized, comprehensive approaches to evaluation, treatment, and monitoring (Lajonchere et al. 2012).

People with ASD and other developmental disorders have the right to attain optimal health, well-being, and functioning and to obtain the highest standard of health care without discrimination (WHO 2013). Children with ASD have the same basic health care needs as children without disabilities and benefit from the same health-promotion and diseaseprevention activities (Myers and Johnson 2007). The most frequently studied topics include genetics, perception and cognition, neurobiology, physiology, and nosology (Matson and LoVullo 2009), with little attention focused on the medical care burden among this group of people. Therefore, it is necessary to examine the health care utilization and expenditure in the provision of medical care to understand the medical care burden of this vulnerable population. The present study reviews the recent literature in Medline, PubMed, and Google by using key terms relevant to ASD and health care (medical care) utilization, medical care costs, and expenditure. I also hand-searched the reference lists of all of the included articles and of recent narratives and systematic reviews related to medical care utilization and the costs for ASD to identify potentially relevant articles.

\section{Medical Care Needs and Utilization}

\section{Medical Needs}

Children with ASD and other developmental disabilities are a subpopulation of the individuals with special health care needs (Association of Maternal and Child Health Programs 2012). There are many major diagnostic challenges in the comprehensive assessment of a child with a suspected ASD, including determining the overall level of functioning, making the categorical diagnosis of an ASD, and identifying the associated etiology (Johnson and Myers 2007). When an ASD case is identified, important medical care for children with ASD includes routine preventive care and the treatment of acute illnesses, management of sleep dysfunction, coexisting challenging behaviors or psychiatric conditions, and associated medical problems, such as seizures, gastrointestinal problems (Myers and Johnson 2007; Lajonchere et al.
2012), auditory disorders and infections (Doshi-Velez et al. 2014), and nutritional deficiencies and metabolic conditions (Lajonchere et al. 2012).

Although there is a marked lack of data on the use of medication treatments for adolescents and young adults with ASD (Dove et al. 2012), a common conceptualization held by many parents is that the more treatments used the better, which has led to many unsubstantiated ASD treatments (Matson et al. 2013). McPheeters et al. (2011) also reported that although many of the children with ASDs are currently treated with medical interventions, strikingly little evidence exists to support the benefit of most of these treatments. Consequently, children with parent-reported ASD had higher levels of health care office visits and prescription drug use compared with children without ASD (Lavelle et al. 2014). In addition, those autistic individuals have a greater probability of having unmet health care needs related to their physical health, mental health, and prescription medications, lower self-reported rates of tetanus vaccination and Papanicolaou smears, and greater likelihood of using the emergency department (Nicolaidis et al. 2012).

Kogan et al. (2008) analyzed the data of the 2005-2006 National Survey of Children with Special Health Care Needs and reported that children with ASD were more likely to have unmet needs for specific health care services, family support services, delayed or foregone care, difficulty receiving referrals, and care that is not family centered. Further analysis by Chiri and Warfield (2012) revealed that the overall unmet need for each type of service ranged from $2.5 \%$ for routine preventive care to $15 \%$ for mental health services, and children with ASD were in fact significantly more at risk for having unmet specialty and therapy care needs. In addition, Parish et al. (2012) analyzed the same data and indicated that compared with White children, Latino children with autism and other developmental disabilities had a consistent pattern of poorer health care access, utilization, and quality.

\section{Medical Care use}

Tregnago and Cheak-Zamora (2012) conducted a systematic review of the literature, confirming that many health care disparities exist between children with ASD and children without ASD, concluding that children with ASD tend to have higher utilization rates of inpatient and outpatient services, emergency services, special services, and pharmaceuticals than children without ASD. An empirical study revealed that children with ASD had a higher annual mean number of total clinic (5.6 vs 2.8 ), pediatric ( 2.3 vs 1.6 ), and psychiatric ( 2.2 vs 0.3 ) outpatient visits than children without ASD. A higher percentage of children with ASD experienced inpatient ( 3 vs $1 \%$ ) and outpatient (5 vs $2 \%$ ) hospitalizations (Croen et al. 2006). 
Autistic adults reported lower satisfaction with patientprovider communication, general health care self-efficacy, and chronic condition self-efficacy (Nicolaidis et al. 2012). Over a third of the parents of children with ASD reported problems when accessing needed specialty care for their child (Krauss et al. 2003). Young et al. (2009) reported that an indirect association was found between the out-of-pocket expense and parents' satisfaction with the services' payer, access to care, and family outcomes in the services for children with ASD. With regard to the perception of medical care providers, primary care physicians expressed that the following barriers to providing primary care were endorsed as greater for children with ASD: lack of care coordination, reimbursement and physician education, families' skepticism about traditional medicine and vaccines, and patients using complementary alternative medicine (Golnik et al. 2009). The above literature analyses highlight that the disparities in the unmet needs of children with ASD suggest that the organizational features of managed care programs and providers' characteristics pose barriers to accessing care (Chiri, and Warfield 2012).

\section{Medical Care Expenditure}

The health care expenditure for children with ASD is substantial and has varied in the past decades; however, the burden of ASD on the health care system will continue to increase (Leslie and Martin 2007). According to 1985-86 data, the average annual health care expenditure for children with ASD was approximately $\$ 1,000$ and approximately $\$ 1,700$ for young adults with ASD compared with an average of $\$ 414$ average for all American children. The children with ASD also visited a physician an average of four times annually, slightly above the US average for all children, and their hospitalization rate was twice the average for all children (Birenbaum et al. 1990). In recent studies, Wang and Leslie (2010) analyzed the reimbursement data for health care expenditures associated with ASD in 42 state Medicaid programs in the USA, finding that the mean total health care expenditure per child with ASD aged 17 years and under was $\$ 22,079$ in 2000 and rose by $3.1 \%$ to $\$ 22,772$ in 2003 . Moreover, Peacock et al. (2012) reported that the mean medical expenditure for Medicaid-enrolled children with ASD was six times higher than for those without ASD. These data indicated that Medicaid-reimbursed health care expenditures for ASD were quite substantial.

In the Kaiser Permanente Medical Care Program in northern California, Croen et al. (2006) analyzed the data and found that the mean annual member costs for hospitalizations ( $\$ 550$ vs $\$ 208)$, clinic visits $(\$ 1,373$ vs $\$ 540)$, and prescription medications ( $\$ 724$ vs \$96) were more than double for children with ASD compared with children without ASD aged 2-18 years. The mean annual age- and gender-adjusted total cost per member was more than threefold higher for children with ASD (\$2,757 vs \$892). In the UK, Lavelle et al. (2014) used national data from the Medical Expenditure Panel Survey linked to the National Health Interview Survey to report that ASD was associated with $\$ 3,020$ higher health care costs and $\$ 14,061$ higher aggregate non-health care costs, including higher school costs of $\$ 8,610$.

In China, Wang et al. (2012) reported that the economic burden of health care for individuals with ASD is substantially higher than the provincial average in Heilongjiang. Families with children with ASD from urban and rural areas had higher per-capita household expenditures of 60.8 and $74.7 \%$, respectively, and in $19.9 \%$ of urban and $38.2 \%$ of rural families, the health care costs exceeded the total annual household income. Our previous study, based on the National Health Insurance claims data in Taiwan, found that the average frequency of hospital admission in the ASD population was 3.5 annually; $47.4 \%$ cases had fewer than two instances of inpatient care, $22.7 \%$ had inpatient care $3-4$ times, $30.0 \%$ were hospitalized more than five times in 2005; and the total inpatient fee was NT\$94,293 (Lin et al. 2011). The autistic individuals with younger ages, serious illnesses, more frequent inpatient care, and longer inpatient stays were more likely to require greater medical expenditures than their counterparts.

\section{ASD Co-occurring Conditions}

Comorbidity is common in individuals with ASD, and more than $70 \%$ have concurrent conditions (Lai et al. 2014). Peacock et al. (2012) found that $47 \%$ of children with ASD had at least one selected co-occurring condition, with attention deficit/hyperactivity disorder (ADHD) the most common, at $30 \%$. Children with ASD and intellectual disability (ID) incurred expenditures 2.7 times higher than did children with ASD and no co-occurring conditions. Lunsky et al. (2009) reported that inpatients with ASD and ID were recommended for a higher level of care than hospital service users without ID. However, the families of children diagnosed with ASD comorbid with ADHD or ID reported the highest levels of dissatisfaction across all health care quality variables and reported the greatest impact on the family's financial situation (Zablotsky et al. 2014).

\section{Mental Health Issues}

Individuals with ASD carry a high risk for coexisting mental health problems from a broad spectrum of disorders as well as unfavorable psychosocial life circumstances (Hofvander et al. 2009). Amr et al. (2012) estimated the prevalence of comorbid psychiatric disorders in a sample of children with ASD recruited from three Arab countries. They indicated that the most commonly reported comorbid disorders were anxiety 
disorders (58.3\%), ADHD (31.6\%), conduct disorders $(23.3 \%)$, and major depressive disorder $(13.3 \%)$. Croen et al. (2006) reported that children with ASD were nearly nine times more likely to use psychotherapeutic medications.

In general, children with ASD are an under-identified and underserved group of children whose behavioral health needs are unmet (Ruble et al. 2005). Behavioral therapy accounted for the largest proportion of health expenses $(54.3 \%)$ for ASD children in China (Wang et al 2012). Our previous study found that ASD cases with ID are more likely to undergo psychiatric visits than their counterparts (Lin et al. 2013). Leslie and Martin (2007) analyzed children and adolescents 17 years and younger with a diagnosis of a mental disorder, and the average health care expenditure for individuals with an ASD increased $20.4 \%$ from $\$ 4,965$ per patient in 2000 to $\$ 5,979$ per patient in 2004, even after adjustment for inflation.

Children with ASD were nearly nine times more likely to use psychotherapeutic medications than children without ASD (Croen et al. 2006). Psychotropic medication use is common among even very young children with ASD, and $56 \%$ used at least one psychotropic medication, $20 \%$ of whom were prescribed three medications (Mandell et al. 2008). Spencer et al. (2013) examined psychotropic medication use and polypharmacy in children with ASD, revealing that $64 \%$ cases had a filled prescription for at least one psychotropic medication, $35 \%$ had evidence of psychotropic polypharmacy ( $\geqq 2$ classes), and $15 \%$ used medications from $\geqq 3$ classes concurrently. Based on the relevant findings, the development standards of care for the prescription of psychotropic medications to children with ASD is necessary (Spencer et al. 2013).

\section{Conclusion}

ASD is an increasingly prevalent diagnosis, and its spectrum of symptoms and treatments are complex (Garrett and Schiff 2013). The UK data showed that the annual costs for children with an ASD and a learning disability who are living in residential or foster placements are estimated to be £16,185-97,863 (aged 0-17) for those living long-term in hospitals (Foundation for People with Learning Disabilities 2007). The average loss of annual income associated with having a child with ASD was $\$ 6,200$, or $14 \%$ of the reported family income (Montes and Halterman 2008). The care burden for ASD was quite substantial.

In medical care service for children with ASD, primary care physicians generally report a lack of self-perceived competency, a desire for education, and a need for improvement in the primary care of children with ASD (Golnik et al. 2009). Parish et al. (2012) suggested that interventions should target improving the providers' cultural sensitivity and behavior during the clinical encounter to reduce the disparities in the health care utilization of children with ASD. All children who are diagnosed with ASD need health care coverage that is universal and continuous, adequate to meet their needs, and affordable (Association of Maternal and Child Health Programs 2012). Continued monitoring is needed to document and understand changes in the condition over time, including the multiple ascertainment and potential risk factors likely to be contributing to children having ASD (CDC 2009).

The literature on medical care utilization and expenditures related to ASD highlights the fact that the disorder imposes high medical burdens on the immediate family as well as on society. This review underscores, as suggested by Zablotsky et al. (2014), that there is a need for comprehensive and accessible medical care services for individuals with ASD, particularly for those with comorbid conditions. Future research is needed to evaluate the impact of improvements in the management of children with ASD on medical care utilization and costs (Croen et al. 2006).

Acknowledgments I am very grateful to Professor Johnny Matson for offering me the opportunity and encouragement for this paper. The author also would like to acknowledge the Republic of China (Taiwan) Ministry of Science and technology for its financial support of this research (grant no. NSC 101-2314-B-016-026-MY3).

\section{References}

Amr, M., Raddad, D., El-Mehesh, F., Bakr, A., Sallam, K., \& Amin, T. (2012). Comorbid psychiatric disorders in Arab children with autism spectrum disorders. Research in Autism Spectrum Disorders, 6, 240-248.

Association of Maternal \& Child Health Programs. (2012). The affordable care act and children and youth with autism spectrum disorder and other developmental disabilities. AMCHP Issue Brief, May, 2012.

Birenbaum, A., Guyot, D., \& Cohen, H. J. (1990). Health care financing for severe developmental disabilities. Monographs of the American Association on Mental Retardation, 14, 1-150.

Centers for Disease Control and Prevention. (2007a). Prevalence of autism spectrum disorders-Autism and Developmental Disabilities Monitoring Network, Six Sites, United States, 2000. MMWR Surveillance Summary, 56 (No.SS-1).

Centers for Disease Control and Prevention. (2007b). Prevalence of autism spectrum disorders-Autism and Developmental Disabilities Monitoring Network, 14 Sites, United States, 2002. MMWR Surveillance Summary, 56 (No.SS-1).

Centers for Disease Control and Prevention. (2009). Prevalence of autism spectrum disorders-Autism and Developmental Disabilities Monitoring Network, United States, 2006. MMWR Surveillance Summary, 58, 1-20.

Centers for Disease Control and Prevention. (2012). Prevalence of autism spectrum disorders-Autism and Developmental Disabilities 
Monitoring Network, United States, 2008. MMWR Surveillance Summary, 61 (No.SS-3).

Chiri, G., \& Warfield, M. E. (2012). Unmet need and problems accessing core health care services for children with autism spectrum disorder. Maternal and Child Health Journal, 16, 1081-1091.

Croen, L. A., Najjar, D. V., Ray, G. T., Lotspeich, L., \& Bernal, P. (2006). A comparison of health care utilization and costs of children with and without autism spectrum disorders in a large group-model health plan. Pediatrics, 118, e1203-e1211.

Curran, A. L., Sharples, P. M., White, C., \& Knapp, M. (2001). Time costs of caring for children with severe disabilities compared with caring for children without disabilities. Developmental Medicine \& Child Neurology, 43, 529-533.

Doshi-Velez, F., Ge, Y., \& Kohane, I. (2014). Comorbidity clusters in autism spectrum disorders: an electronic health record time-series analysis. Pediatrics, 133, e54-e63.

Dove, D., Warren, Z., McPheeters, M. L., Taylor, J. L., Sathe, N. A., \& Weele, J. V. (2012). Medications for adolescents and young adults with autism spectrum disorders: a systematic review. Pediatrics, 130, 717-726.

Foundation for People with Learning Disabilities. (2007). The economic consequences of autism in the UK. London: Executive research and policy briefing from the Foundation for People with Learning Disabilities.

Garrett, E., \& Schiff, J. S. (2013). Autism spectrum disorders. Report to the Minnesota Commissioner of Human Services Advisory Council. Minnesota Department of Human Services, February 2013.

Golnik, A., Ireland, M., \& Borowsky, I. W. (2009). Medical homes for children with autism: a physician survey. Pediatrics, 123, 966-971.

Hofvander, B., Delorme, R., Chaste, P., Nydén, A., Wentz, E., \& Ståhlberg, O. (2009). Psychiatric and psychosocial problems in adults with normal-intelligence autism spectrum disorders. $B M C$ Psychiatry, 9, 35

Johnson, C. P., \& Myers, S. M. (2007). Identification and evaluation of children with autism spectrum disorders. Pediatrics, 120, 1183-1215.

Kanner, L. (1943). Autistic disturbances of affective contact. The Nervous Child, 2, 217-250.

Kogan, M. D., Strickland, B. B., Blumberg, S. J., Singh, G. K., Perrin, J. M., \& van Dyck, P. C. (2008). A national profile of the health care experiences and family impact of autism spectrum disorder among children in the United States, 2005-2006. Pediatrics, 122, e1149-e1158.

Kogan, M. D., Blumberg, S. J., Schieve, L. A., Boyle, C. A., Perrin, J. M., Ghandour, R. M., et al. (2009). Prevalence of parent-reported diagnosis of autism spectrum disorder among children in the US, 2007. Pediatrics, 124, 1395-1403.

Krauss, M. W., Gulley, S., Sciegaj, M., \& Wells, N. (2003). Access to specialty medical care for children with mental retardation, autism, and other special health care needs. Mental Retardation, 41, 329-339.

Lai, M. C., Lombardo, M. V., \& Baron-Cohen, S. (2014). Autism. Lancet, 383, 896-910.

Lajonchere, C., Jones, N., Coury, D. L., \& Perrin, J. M. (2012). Leadership in health care, research, and quality improvement for children and adolescents with autism spectrum disorders: Autism Treatment Network and Autism Intervention Research Network on Physical Health. Pediatrics, 130, S62-S68.

Lavelle, T. A., Weinstein, M. C., Newhouse, J. P., Munir, K., Kuhlthau, K. A., \& Prosser, L. A. (2014). Economic burden of childhood autism spectrum disorders. Pediatrics, 133, e520-e529.

Leslie, D. L., \& Martin, A. (2007). Health care expenditures associated with autism spectrum disorders. Archives of Pediatrics \& Adolescent Medicine, 161, 350-355.
Lin, J. D., Hung, W. J., Lin, L. P., \& Lai, C. I. (2011). Utilization and expenditure of hospital admission in patients with autism spectrum disorder: National Health Insurance claims database analysis. Research in Autism Spectrum Disorders, 5, 1138-1142.

Lin, L. P., Kuan, C. Y., Hsu, S. W., Lee, T. N., Lai, C. I., Wu, J. L., et al. (2013). Outpatient visits and expenditures for children and adolescents diagnosed with autism spectrum disorders and co-occurring intellectual disability: an analysis of the national health insurance claims data. Research in Autism Spectrum Disorders, 7, 1625-1630.

Lunsky, Y., Gracey, C., \& Bradley, E. (2009). Adults with autism spectrum disorders using psychiatric hospitals in Ontario: clinical profile and service needs. Research in Autism Spectrum Disorders, 3, 1006-1013.

Maenner, M. J., \& Durkin, M. S. (2010). Trends in the prevalence of autism on the basis of special education data. Pediatrics, 126 , e1018-e1025.

Mandell, D. S., Morales, K. H., Marcus, S. C., Stahmer, A. C., Doshi, J., \& Polsky, D. E. (2008). Psychotropic medication use among Medicaid-enrolled children with autism spectrum disorders. Pediatrics, 121, e441-e448.

Matson, J. L., \& LoVullo, S. V. (2009). Trends and topics in autism spectrum disorders research. Research in Autism Spectrum Disorders, 3, 252-257.

Matson, J. L., Adams, H. L., Williams, L. W., \& Rieske, R. D. (2013). Why are there so many unsubstantiated treatments in autism? Research in Autism Spectrum Disorders, 7, 466- 474.

McPheeters, M. L., Warren, Z., Sathe, N., Bruzek, J. L., Krishnaswami, S., \& Jerome, R. N. (2011). A systematic review of medical treatments for children with autism spectrum disorders. Pediatrics, 127, e1312-e1321.

Montes, G., \& Halterman, J. S. (2008). Association of childhood autism spectrum disorders and loss of family income. Pediatrics, 121, e821-e826.

Muhle, R., Trentacoste, S. V., \& Rapin, I. (2004). The genetics of autism. Pediatrics, 113, e472-e486.

Myers, S. M., \& Johnson, C. P. (2007). Management of children with autism spectrum disorders. Pediatrics, 120, 1162-1182.

Nicolaidis, C., Raymaker, D., McDonald, K., Dern, S., Boisclair, W. C., Ashkenazy, E., et al. (2012). Comparison of healthcare experiences in autistic and non-autistic adults: a cross-sectional online survey facilitated by an academiccommunity partnership. Journal of General Internal Medicine, 28, 761-769.

Parish, S., Magaña, S., Rose, R., Timberlake, M., \& Swaine, J. G. (2012). Health care of Latino children with autism and other developmental disabilities: quality of provider interaction mediates utilization. American Journal on Intellectual and Developmental Disabilities, $117,304-315$.

Peacock, G., Amendah, D., Ouyang, L., \& Grosse, S. D. (2012). Autism spectrum disorders and health care expenditures: the effects of cooccurring conditions. Journal of Developmental Behavioral Pediatrics, 33, 2-8.

Rice, C., Schendel, D., Cunniff, C., \& Doernberg, N. (2004) Public health monitoring of developmental disabilities with a focus on the autism spectrum disorders. American Journal of Medical Genetics Part C Seminar in Medical Genetics, $125 C, 22-27$.

Ruble, L. A., Heflinger, C. A., Renfrew, J. W., \& Saunders, R. C. (2005). Access and service use by children with autism spectrum disorders in Medicaid managed care. Journal of Autism and Developmental Disorders, 35, 3-13.

Spencer, D., Marshall, J., Post, B., Kulakodlu, M., Newschaffer, C., Dennen, T., et al. (2013). Psychotropic medication use and polypharmacy in children with autism spectrum disorders. Pediatrics, 132, 833-840. 
Tregnago, M. K., \& Cheak-Zamora, N. C. (2012). Systematic review of disparities in health care for individuals with autism spectrum disorders in the United States. Research in Autism Spectrum Disorders, 6, 1023-1031.

Wang, L., \& Leslie, D. L. (2010). Health care expenditures for children with autism spectrum disorders in Medicaid. Journal of American Academy of Child and Adolescent Psychiatry, 49, 1165-1171.

Wang, J., Zhou, X., Xia, W., Sun, C. H., Wu, L. J., Wang, J. L., et al. (2012). Parent-reported health care expenditures associated with autism spectrum disorders in Heilongjiang province, China. $B M C$ Health Services Research, 12, 7.
WHO. (2013). Autism spectrum disorders \& other developmental disorders-from raising awareness to building capacity. Geneva: Meeting Report of World Health Organization.

Young, A., Ruble, L., \& McGrew, J. (2009). Public vs. private insurance: cost, use, accessibility, and outcomes of services for children with autism spectrum disorders. Research in Autism Spectrum Disorders, 3, 1023-1033.

Zablotsky, B., Kalb, L. G., Freedman, B., Vasa, R., \& Stuart, E. A. (2014). Health care experiences and perceived financial impact among families of children with an autism spectrum disorder. Psychiatric Services, 65, 395-398. 